\title{
Partial Resummation of $\hbar$-Expansion of the Bloch Density for non Local Potentials
}

\author{
M. Durand \\ Institut des Sciences Nucléaires, Grenoble, France \\ P. Schuck \\ Institut Laue-Langevin, Grenoble, France \\ M. Brack \\ Institut für Theoretische Physik, Universität Regensburg, Regensburg, \\ Federal Republic of Germany
}

Received March 20, 1980

\begin{abstract}
The Wigner-Kirkwood $\hbar$-expansion of theWigner transform of the Bloch density can be resummed in the case of non local potentials if we keep only up to second order derivatives of the Wigner transform of the non-local potential with respect to the phase space variables. We also investigate a second approximation to the Bloch density where care has been taken with respect to a consistent $\hbar$ expansion. For a one dimensional example we calculate the smooth part of the density and the corresponding energy demonstrating that both approximations to the Bloch-density yield well defined average densities and energies.
\end{abstract}

\section{Introduction}

Semi classical $\hbar$-expansion based on the WignerKirkwood method yield the Strutinsky values for the average binding energies but the corresponding density is not well defined close to the classical turning point. This difficulty with the classical turning point has recently been overcome $[4,5]$ by partial resummation of the $\hbar$-expansion leading to densities with very satisfactory surface behaviour through the entire region of interest.

Realistic Hartree-Fock theory also requires the treatment of non local single particle potentials. It is the purpose of this paper to generalize the technique of partial resummation of the $\hbar$-expansion to non local potentials. As in the case of local potentials we achieve a resummation of the Wigner Kirkwood series [5] for non local potentials involving all first and second order derivatives of the potential with respect to the phase space variables. In addition we also give in this paper a form of the propagator which yields well defined densities in the region of the classical turning point but remains a consistent $h$-expansion of func- tions simply related to the Bloch density. A numerical example is given which demonstrates that our methods work very well also in the non-local case.

\section{Partial Resummation of $\hbar$-Expansion}

As in the case of local potentials [5] we start with the Bloch density matrix

$C_{\mathbf{r r}}^{\beta}=\left\langle\mathbf{r}|\exp (-\beta H)| \mathbf{r}^{\prime}\right\rangle$

where $\beta=\mathrm{it} / \hbar$ and $H$ a single particle Hamiltonian. The single particle density matrix is then given by an inverse Laplace transformation [6]:

$\rho\left(\mathbf{r}, \mathbf{r}^{\prime}\right)=\mathscr{L}_{\beta \rightarrow \mu}^{-1}\left[\frac{1}{\beta} C_{\mathbf{r r}^{\prime}}^{\beta}\right]$

where $\mu$ is the chemical potential. Deriving (1) with respect to $\beta$ and Wigner transforming the resulting equation yields $[7,8]$ (for simplicity we restrict ourselves here to the one dimensional case but every- 
thing can be generalized to three dimensions):

$\left\{\frac{\partial}{\partial \beta}+H(R, p) \cos \frac{\hbar}{2} \overleftrightarrow{A}\right\} C_{R, p}^{\beta}=0$

with

$\overleftrightarrow{A}=\frac{\stackrel{\partial}{\partial R}}{\partial \frac{\partial}{\partial p}}-\frac{\grave{\partial}}{\partial p} \vec{\partial}$

where the arrows indicate whether the derivatives shall act to the right or left. The definition of the Wigner transform of an operator $A$ is given by

$\left\langle x|A| x^{\prime}\right\rangle_{W} \equiv A(R, p)$

$=\int_{-\infty}^{+\infty} d s \exp \left(-\frac{i p s}{\hbar}\right)\left\langle R+\frac{s}{2}|A| R-\frac{s}{2}\right\rangle$.

For the Hamiltonian operator we therefore obtain

$H(R, p)=\frac{p^{2}}{2 m}+V(R, p)$

where $V(R, p)$ is the Wigner transform of the non local potential $V\left(x, x^{\prime}\right)$.

In order to achieve the partial resummation of all first and second order derivatives of the potential with respect to the phase space variables $R$ and $p$ figuring in the Wigner-Kirkwood expansion of $C$ [1$3,11]$ we apply the following procedure: we develop the Hamilton function $H(R, p)$ about an arbitrary point $\left(R_{0}, p_{0}\right)$ in phase space to second order:

$$
\begin{aligned}
& H(R, p) \simeq H^{0}+H_{R}^{0}\left(R-R_{0}\right)+H_{p}^{0}\left(p-p_{0}\right) \\
& +\frac{1}{2}\left[H_{R R}^{0}\left(R-R_{0}\right)^{2}+2 H_{R p}^{0}\left(p-p_{0}\right)\left(R-R_{0}\right)\right. \\
& \left.+H_{p p}^{0}\left(p-p_{0}\right)^{2}\right]
\end{aligned}
$$

where lower indices mean derivation and the zero indicates that the function is to be taken at $\left(R_{0}, p_{0}\right)$. Equation (7) represents the Hamilton function of a harmonic oscillator which can be cast into normal form by a linear transformation $(R, p) \leftrightarrow\left(\xi_{1}, \xi_{2}\right)$ :

$H=\tilde{H}^{0}+\frac{\lambda_{1}}{2} \xi_{1}^{2}+\frac{\lambda_{2}}{2} \xi_{2}^{2}$

with

$$
\begin{aligned}
& \tilde{H}^{0}=H^{0}-\frac{1}{2 D^{0}}\left(H_{R}^{0} D_{R}^{0}+H_{p}^{0} D_{p}^{0}\right) \\
& D^{0}=\left|\begin{array}{ll}
H_{R R}^{0} & H_{R p}^{0} \\
H_{R p}^{0} & H_{p p}^{0}
\end{array}\right| D_{R}^{0}=\left|\begin{array}{ll}
H_{R}^{0} & H_{R p}^{0} \\
H_{p}^{0} & H_{p p}^{0}
\end{array}\right| D_{p}^{0}=\left|\begin{array}{ll}
H_{R R}^{0} & H_{R}^{0} \\
H_{R p}^{0} & H_{p}^{0}
\end{array}\right| \\
& \lambda_{1,2}=\frac{1}{2}\left(H_{R R}^{0}+H_{p p}^{0}\right) \pm \frac{1}{2}\left[\left(H_{R R}^{0}-H_{p p}^{0}\right)^{2}+4\left(H_{R p}\right)^{2}\right]
\end{aligned}
$$

Since the form (4) of the operator $\overleftrightarrow{A}$ is invariant under a linear transformation, (3) together with (8) is an equation for a linear harmonic oscillator, the Bloch density of which is known analytically [9].

The approximation depends parametrically on $\left(R_{0}, p_{0}\right)$ and will be best for $(R, p)$ values close to $\left(R_{0}, p_{0}\right)$. We therefore take the limit $(R, p) \rightarrow\left(R_{0}, p_{0}\right)$ and since $\left(R_{0}, p_{0}\right)$ were arbitrary we obtain:

$$
\begin{aligned}
& C_{R, p}^{\beta}=\operatorname{Cosh}^{-1}\left(\frac{\hbar \omega^{\beta}}{2}\right) \exp \{-\beta H(R, p)+X(R, p) \\
& \left.\times\left[\frac{b}{2}-\frac{\operatorname{Tgh}(\hbar \omega \beta / 2)}{\hbar \omega}\right]\right\}
\end{aligned}
$$

with

$\omega=\left(\lambda_{1} \lambda_{2}\right)^{1 / 2}=D^{1 / 2} \quad X=\lambda_{1} \xi_{1}^{2}+\lambda_{2} \xi_{2}^{2}$

This is the form of the Bloch density where we locally harmonized a nonlocal potential in phase space. The Wigner Kirkwood expansion for the propagator (1) is a simultaneous expansion in powers of $\hbar$ and $\beta$. We can therefore recover this expansion apart from third and higher derivatives in expanding (11) in powers of $\beta$. This shows that our procedure for the determination of (11) is correct.

One may object against the form (11) of the propagator that it is not consistent in powers of $\hbar$, that is, the Wigner-Kirkwood series corresponding to (11) is correct up to $\hbar^{2}$ but in the term $\hbar^{4}$, for example, the third and fourth derivatives of the potential are missing. It is however known [10] that in the term $\hbar^{4}$ important cancellations between the different terms are taking place and that individual terms may be big.

We may here therefore try a different but in itself still consistent $\hbar$ expansion. From (11) we remark that the propagator can be decomposed (this holds true in general for any Bloch density):

$C_{R, p}^{\beta}=A(\beta) \exp [-F(\beta)]$

where $A$ is even and $F$ odd in $\beta$. We now can make a systematic Taylor expansion in powers of $\hbar$ for $A$ and $F$ independently.

This is very easily achieved using (11) and we obtain to order $\hbar^{2}$ :

$$
\begin{aligned}
& \tilde{C}_{R, p}^{(2)}=\left(1-\frac{\hbar^{2} \omega^{2}}{8} \beta^{2}\right) \\
& \left.\times \exp \left[-\beta H(R, p)+\beta^{3} \frac{\hbar^{2} \omega^{2}}{24} X(R, p)\right)\right]
\end{aligned}
$$

Since the Bloch denisty is apparently a very fast converging series in $\hbar[10,11]$, it may well be that also $A$ and $F$ independently are rapidly converging functions in powers of $\hbar$. There are indeed indications 
[12] that such a modified $\hbar$-expansion (14) yields better results than forms of the propagator which have not a consistent $h$-expansion built in in some way.

\section{Numerical Example}

For a definite example we want to calculate the local density

$\rho(R)=\frac{1}{2 \pi \hbar} \frac{1}{2 i \pi} \int_{-\infty}^{+\infty} d p \int_{c-i \infty}^{c+i \infty} d \beta \frac{C_{R, p}^{\beta}}{\beta} \exp (\mu \beta)$

and the groundstate energy, corresponding to the sum of the occupied levels

$E=\mu \int_{-\infty}^{+\infty} d R \rho(R)$

$-\frac{1}{2 \pi \hbar} \frac{1}{2 i \pi} \int_{-\infty}^{+\infty} d R \int_{-\infty}^{+\infty} d p \int_{c-i \infty}^{c+i \infty} d \beta \frac{C_{R, p}^{\beta}}{\beta^{2}} \exp (\mu \beta)$.

As was discussed in a preceeding publication [5] we have to average out spurious oscillations in this density taking the inverse Laplace transform as indicated in (2) and (15). In [5] this was done in performing a local Strutinsky smoothing of the propagator. In a forthcoming publication [12] it will be shown that the smoothing can be very efficiently achieved in performing the Laplace inversion with the saddle point method [4]:

$$
\begin{aligned}
& G(\mu)=\frac{1}{2 i \mu} \int_{c-i \infty}^{c+i \infty} d \beta I(\beta) \exp (\mu \beta) \\
& =\frac{1}{2 i \pi} \int_{c-i \infty}^{c+i \infty} d \beta \exp [S(\beta)] \\
& \simeq \frac{\exp \left(S_{0}\right)}{\left(2 \pi \ddot{S}_{0}\right)^{1 / 2}}\left[1+\frac{1}{8 \ddot{S}_{0}^{3}}\left(\dddot{S}_{0} \ddot{S}_{0}-\frac{5}{3} \dddot{S}_{0}^{2}\right)+\ldots\right]
\end{aligned}
$$

where the index zero means that the quantity is taken at the stationary point $(\partial S / \partial \beta)_{0} \equiv \dot{S}_{0}=0$ and approximation (17) is obtained in expanding $S$ around the stationary point $S=S_{0}+\frac{1}{2} \ddot{S}_{0}\left(\beta-\beta_{0}\right)^{2}+\frac{1}{6} \dddot{S}_{0}\left(\beta-\beta_{c}\right)^{3}$ $+\ldots$ and in expanding exp $(S)$ in powers of $\ddot{S}, \dddot{S}, \ldots$ which yields the correction terms. The first order correction is explicitly given in (17) but to obtain a convergent it is usually necessary to include also the second order correction [12].

For non local potentials $(11,14)$ are in general complicated functions of $p$ and the $p$-integrals of the inverse Wigner (Fourier) transform for the calculation of $\rho\left(x, x^{\prime}\right)$ have to be done numerically. For the example considered below it turns out however that a two dimensional generalization of the saddle point method (17) does equally well, which of course makes the task much easier. The search for the two dimensional saddle point is further facilitated by the fact that for time even spin independent potentials the saddle in $p$ is always at $p=0$.

As an example we consider the following form of a non local Hamiltonian

$$
\begin{aligned}
& H\left(x, x^{\prime}\right)=\left(-\frac{\hbar^{2}}{2 m} \frac{\partial^{2}}{\partial x^{2}}+\frac{1}{2} m \omega^{2} x^{2}\right) \delta\left(x-x^{\prime}\right) \\
& +V_{0}\left(\frac{b}{\pi}\right)^{1 / 2} \exp \left[-a\left(x+x^{\prime}\right)^{2}-b\left(x-x^{\prime}\right)^{2}\right]
\end{aligned}
$$

As the width $b^{-1 / 2}$ of the non locality goes to zero the non local potential goes over into a local one which we will treat at the same time in order to study the influence of the non locality. For convenience we choose the constants $a$ and $b$ in such a way that the Wigner transform of the non local part is of the form

$V(R, p) \sim \exp \left[-h^{H o}(R, p) / a\right]$

where $h^{H O}$ corresponds to the harmonic oscillator figuring in (18). In this case (19) is trivially diagonalized since the off diagonal matrix elements are zero.

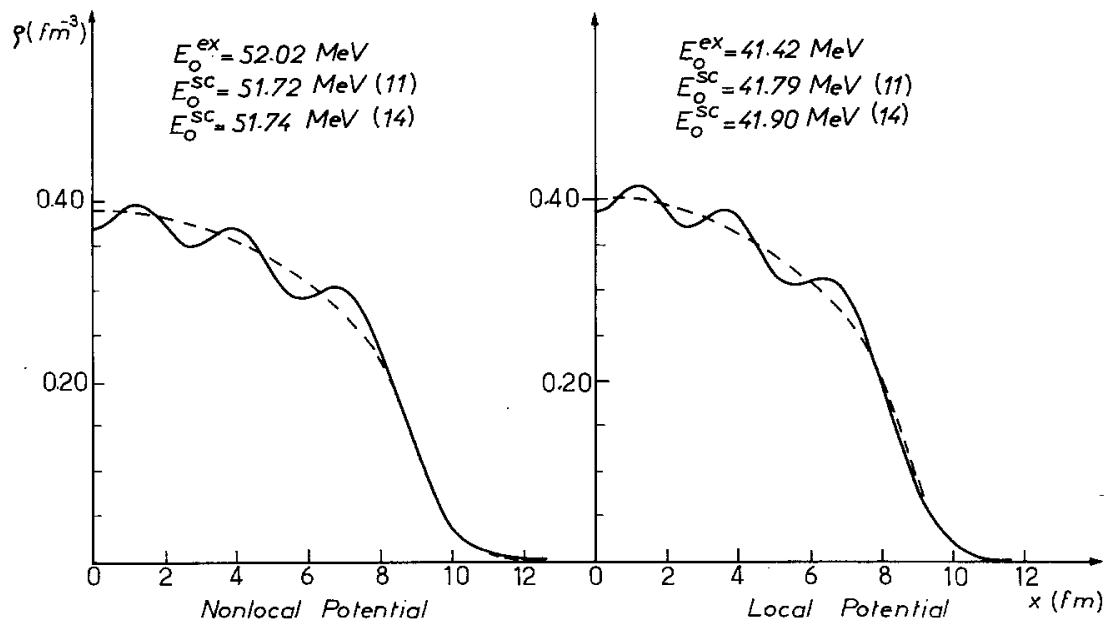

Fig. 1. Exact (solid lines) and semi classical densities (dashed lines) for the non local potential (left) and the corresponding local potential (right). The densities corresponding to Eqs. (11) and (14) are indistinguishable on the figure; the corresponding energy values have been marked with the number of the formula 
The local limit $(b \rightarrow \infty)$ is however diagonalized numerically. For our example we choose the following parameter values:

$V_{0}=-10 \mathrm{MeV} ; \quad \alpha=30 \mathrm{MeV} ; \quad \hbar \omega=5 \mathrm{MeV}$

for a number of particles $A=12$.

We calculated with the methods outlined above (two dimensional saddle point) the average densities and energies for the nonlocal and local cases and the results are displayed in Fig. 1. We notice that the results are in our example practically the same using (11) or (14) for the propagator. We remark that the influence of the non locality in our example changes the values for the groundstate energy and the density by about $20 \%$ which is a realistic effect compared with physical situations. More precisely we see for the density that the non locality lowers the density in the interior and pushes it out to higher $R$-values, an effect which is reproduced by our semi classical method. As a matter of fact our density follows the exact one very nicely on the average and passes for instance smoothly through the classical turning point; that would not have been the case if we had used the pure Wigner-Kirkwood expansion. Also the semi classical groundstate energies agree within an error of per thousands with the exact ones which is typical for the Strutinsky method or semi classical values [13]. In a forthcoming publication we will investigate the more realistic non localities of the Skyrme type.

\section{Conclusion}

In this work we investigated the generalization of the partial $h$-resummation technique $[4,5]$ to non local potentials. It thereby turned out that a locally harmonic approximation of the non local potential in phase space yields, together with the saddle point method for the Laplace inversions involved, very sensible results for average quantities such as total energy, spatial density etc. Besides the locally harmonic approximation we here also proposed a form of the propagator with an inherent consistent $\hbar$ expansion. This was achieved in dividing the propagator in a certain way into a $\beta$-even and a $\beta$-odd part and expanding these parts independently into powers of $\hbar$. The two approximations for the Bloch density give practically the same results for our numerical example. It then becomes clear that the technique of partial $\hbar$-resummation can be successfully applied to cases where non local potentials are involved such as the exchange part of the HartreeFock potential. This will of course be important for the semi classical approximation to a fully self consistent calculation. Investigations in this direction are under way.

We appreciated useful information and interest in this work by $D$. Sprung.

\section{References}

1. Wigner, E.: Phys. Rev. 40, 749 (1932)

2. Kirkwood, J.G.: Phys. Rev. 44, 31 (1933)

3. Imre, K., Ozizmir, E., Rosenbaum, M., Zweifel, P.F.: J. Math. Phys. 8, 1097 (1967)

4. Bhaduri, R.K.: Phys. Rev. Lett. 39, 329 (1977)

5. Durand, M., Brack, M., Schuck, P.: Z. Physik A286, 381 (1978)

6. Sondheimer, E.H., Wilson, A.H.: Proc. Roy. Soc. A210, 173 (1951)

7. Gronewold, H.J.: Physica 12, 405 (1946)

8. Theis, W.R.: Z. Physik 142, 503 (1955)

9. Gol'dman, Krivchenkov, Kogan, Galitzkii: Selected problems in quantum mechanics. ter Haar. D. (ed.), Infosearch Ltd (London) (1964) Sect. (3.24)

10. Jennings, B.K.: Ph. D. Thesis, Mc Master University, 1976

11. Jennings, B.K., Bhaduri, R.K., Brack, M.: Nucl. Phys. A253, 29 (1975)

12. Bartel, J., Schuck, P., Durand, M., Bhaduri, R.K., Brack, M.: to be published

13. Brack, M., Pauli, H.C.: Nucl. Phys. A207, 401 (1973)

M. Durand

Institut des Sciences Nucléaires

(IN2P3-USMG)

F-38026 Grenoble-Cedex

France

P. Schuck

Institut Max von Laue - Paul Langevin

Avenue des Martyrs

$156 \mathrm{X}$

F-38042 Grenoble Cedex

France

M. Brack

Institut für Theoretische Physik

Universität Regensburg

Universitätsstraße 31

D-8400 Regensburg

Federal Republic of Germany 